\title{
Traitement de la Trichostrongylose humaine par l'hydroxynaphtoate de Bephenium (Alcopar)
}

\author{
par Esmaïl GHADIRIAN et Hossein BIJAN \\ Faculté d'Hygiène et Institut de Recherches de la Santé publique \\ de PUniversité de Téhéran (Iran)
}

\begin{abstract}
Résumé
85 malades furent traités par l'Alcopar. 20 d'entre eux furent complètement et définitivement guéris.
\end{abstract}

\begin{abstract}
Summary
85 patients were treated with Alcopar. 20 of them, were completely and definitively cured.
\end{abstract}

\section{Introduction.}

Les infestations humaines à Trichostrongylus sont connues depuis 1949 en Iran (1-2), mais la répartition géographique selon les différentes régions n'avait pas été précisée. Récemment, l'Institut de Recherches de la Santé Publique a pu déterminer avec précision le pourcentage d'infestation dans certaines régions d'Iran et constater ainsi qu'elle pouvait dépasser $69 \%$ de la population (3) à Ispahan ; par contre, dans la région

Les auteurs tiennent à remercier le Professeur Ch. Mofidi, Directeur de la Faculté d'Hygiène et de l'Institut de Santé Publique, de ses précieux conseils, ainsi que les Docteurs R. Sabokbar, A. Nadim, A. Sanati, de leur aide efficace.

* Ces études ont été financées par la Faculté d'Hygiène (Université de Téhéran et Institut de Recherches de la Santé Publique (Organisation du Plan).

Annales de Parasitologie humaine et comparée (Paris), t. 43, 1968, $\mathrm{n}^{\circ} 4$ 
du Khuzistan (sud de l'Iran près du Golfe Persique). Le taux varie de 60 à $66 \%$ (4-5) pour s'abaisser à $7,5 \%$ sur le bord de la mer Caspienne (5) et $42 \%$ pour Téhéran (4).

Ce parasite est considéré comme pathogène par certains auteurs (10), il peut déterminer des troubles digestifs et même causer une légère anémie, ce qui nous a conduit à étudier les modalités de son traitement. Par analogie avec la thérapeutique de l'ankylostomiase, infection également à localisation duodénale, nous avons choisi l'Alcopar comme traitement de la trichostrongylose humaine.

\section{Mode du traitement :}

Sur 1.045 examens de selles, nous avons sélectionné 85 cas de fortes infestations permettant de contrôler facilement l'effet de la thérapeutique. Nous avons utilisé l'Alcopar* (7-8).

Les malades sont mis à la diète lactée la veille du traitement, la dose thérapeutique est administrée en une seule fois, à jeun; dans un verre d'eau froide, on donne un sachet de $5 \mathrm{~g}$ pour les sujets âgés de plus de 7 ans, et un sachet de $2,50 \mathrm{~g}$ pour les enfants de 2 à 7 ans. Nous n'avons pas traité d'enfants âgés de moins de 2 ans. Après la prise du médicament, le malade est laissé au repos et un purgatif salin lui est administré trois heures après. Lorsque la prise de l'Alcopar entraîne des nausées ou des vomissements, nous prescrivons $10 \mathrm{cg}$ de Luminal (Phenolbarbital) ou $25 \mathrm{mg}$ de Largactil (Chlorpromazine).

Les matières fécales sont examinées par la méthode de flottation; $2 \mathrm{~g}$ de fèces sont délayés dans $32 \mathrm{ml}$ d'une solution saturée de chlorure de sodium; la durée de la flottation est de 15 minutes; puis on compte le nombre d'œufs de Trichostrongylus; cette opération est répétée quatre fois pour chaque examen de selles, le chiffre moyen est adopté. Parallèlement, on compte le nombre d'œufs par la méthode de Stoll, également répétée quatre fois.

Le premier examen est pratiqué à la veille du traitement, le deuxième, trois jours après le traitement, puis on fait des examens tous les dix jours pendant un mois.

\section{Résultats :}

Sur 85 malades traités, on a observé les résultats suivants :

- Dans 20 cas $(23,5 \%)$, la guérison fut complète dès le premier examen et s'est maintenue aux examens suivants.

- Dans 65 autres cas, où les contrôles ont pu également être pratiqués régulièrement, la stérilisation n'a pas été complète, mais la numération des œufs a montré une diminution très importante de leur nombre, comme on peut le voir sur le tableau ci-contre.

**a Fabriqué par les laboratoires Wellcome en sachets de 5 grammes comprenant chacun 2,50 grammes de Bephenium. 


\section{Tableau}

RÉSULTAT DU TRAITEMENT DE LA TRICHOSTRONGYLOSE SUR 85 SUJETS INFESTÉS

\begin{tabular}{|c|c|c|c|c|c|}
\hline & \multicolumn{5}{|c|}{ Nombre moyen des œufs chez chaque sujet infesté } \\
\hline $\begin{array}{l}\text { Nombre de } \\
\text { sujets infestés }\end{array}$ & Avant le & \multicolumn{4}{|c|}{ Après le traitement } \\
\hline 20 complètement & & $1^{\text {er }}$ examen & $2^{\circ}$ examen & $3^{\mathrm{e}}$ examen & $4^{e}$ examen \\
\hline guéris $\ldots \ldots \ldots \ldots$ & 32 & 0 & 0 & 0 & 0 \\
\hline $\begin{array}{l}65 \text { partiellement } \\
\text { guéris } \quad \ldots \ldots \ldots \ldots\end{array}$ & 30 & 2 & 1,5 & 1 & 1 \\
\hline
\end{tabular}

\section{Bibliographie}

1. Ansari (N.), 1950. - Parasitologie médicale, V. 2 P., 194, Faculté de Médecine de Téhéran (en langue persane).

2. Bilharziasis Report, July, 1963. - Inst. of P.H.R. Téhéran Publication, No. 1198.

3. EISA (A. M.) et RoBIN (R.), 1960. - Bephenium hydroxynaphtoate ; a new and promising gastro intestinal anthelmintic for cattle. Journal of Parasitology, 46 (5 sect. 2), 8.

4. Ghadirian (A.), Mofidi (Ch.) et BiJan (H.) (sous presse), - Premiers travaux sur l'identification des différentes espèces de Trichostrongylus en Iran. $1^{\text {re }}$ partie: étude des Trichostrongylus des régions de Téhéran, Ispahan et du Khuzestan.

5. Ghadirian (A.), Bijan (H.) (sous presse). - Premiers travaux sur l'identification de différentes espèces de Trichostrongylus en Iran. $2^{\circ}$ partie : étude sur les Trichostrongylus faites dans le nord de l'Iran.

6. Hahn (S.S.), Kang (H. Y.) and Hahn (Y.S.), 1960. - The anthelmintic effect to bephenium hydroxynaphtoate on intestinal Helminthe. J. Trop. Med. and Hyg., Aug. V, 63-No., 8, P., 180-184.

7. Otsuru (M.) and Hasegawa (K.), 1961. - The anthelmintic effect of bephenium hydroxynaphtoate (Alcopar against Trichostrongylus). Japanese Journal of Parasitology, 10, (5), 617-621.

8. Rawes (D. A.) and Scarnell (J.), 1959. - Bephenium hydroxynaphtoate. Its activity on Nematodirus and other Trichostrongylus nematods in lambs: Veterinary Record, 7.1 (31), 645-650.

9. Stewart (I. S.), 1949. - Human infestation with Trichostrongylus in South Persia. Brit. Med. J., 1949, Oct., 1, 73-78.

10. Watson (J. M.), 1960. - Medical Helminthology: Trichostrongylidae. P. 153. London, Baillière Tindall and Cox. 